


\section{REVISTA DE PSICOLOGÍA}

Vol. XXIII. Segundo semestre 2005 Nº 2

\section{CONTENIDO}

\section{ARTÍCULOS}

Denise Benatuil, Alejandro Castro Solano y Alejandro Torres. Inteligencia práctica: un instrumento para su evaluación.

Dora Herrera Paredes y Hugo Morales Córdova. Comportamiento antisocial durante la adolescencia: teoría, investigación y programas de prevención.

James S. Fleming y César Merino Soto. Medidas de simplicidad y de ajuste factorial: un enfoque para la evaluación de escalas construidas factorialmente.

Cecilia Chau y Stephan Van den Broucke. Consumo de alcohol y sus determinantes en estudiantes universitarios limeños: estudio de focus group.

Inés Kudó, Tesania Velásquez, Mónica Iza, Alicia Ángeles, César Pezo del Pino y Patricia Martínez U. Una experiencia de intervención en crisis en el sur del Perú: Brigadas Psicológicas. 
Revista de Psicología de la PUCP. Vol. XXIII, 2, 2005

\title{
Medidas de simplicidad y de ajuste factorial: un enfoque para la evaluación de escalas construidas factorialmente
}

\author{
James S. Fleming ${ }^{1}$ \\ Embry-Riddle Aeronautical University, Northern Arizona University \\ César Merino Soto ${ }^{2}$ \\ Defensoría Municipal del Niño y del Adolescente
}

\begin{abstract}
Una estructura simple es sólida cuando se utiliza el análisis factorial para desarrollar instrumentos de medición. El presente artículo se basa en el programa SIMLOAD, que produce índices que miden la simplicidad factorial para las filas y columnas de una matriz de cargas factoriales (generalmente, el patrón de factores), tanto como índices globales de ajuste. Estos incluyen para las filas el índice de simplicidad factorial de Kaiser (1974); para las columnas, el índice de ajuste a la escala de Fleming; la medida matriz libre de escalas de Bentler (1977); y, el conteo de hiperplanos. Estas medidas se recomiendan para la construcción de escalas multifactoriales. Estos índices también pueden ser útiles para aplicaciones factoriales más generales, en el análisis exploratorio o confirmatorio. El programa SIMLOAD también calcula intercorrelaciones entre las escalas, coeficientes alfa de Cronbach, el efecto de la eliminación de un ítem sobre el alfa y el ordenamiento de las cargas factoriales de los ítems para facilitar la interpretación de la matriz resultante.

Palabras clave: análisis factorial, escalas, investigación psicométrica.
\end{abstract}

Measures of simplicity and factorial fit: An approach for the evaluation of factorially derived scales

A very simple structure is sought when using factor analysis to develop measurement scales. The present article is about the SIMLOAD program; it computes measures of factor simplicity for rows and columns of loading matrices (usually the factor pattern) as well as some overall measures. These include Kaiser's (1974) index of factor simplicity for variables (rows), Fleming's scale fit index for factors (columns), Bentler's (1977) scale-free matrix measure, and hyperplane counts. Routine use of these measures is recommended for multifactor scale development. The measures may also be useful in more general factor applications, and in confirmatory as well as exploratory analysis. SIMLOAD additionally computes factor scale intercorrelations, scale alpha coefficients, including alpha when item removed, and sorted loadings for ease of interpretation.

Keywords: factor analysis, scales, psychometric research.

1 Doctor en Psicología. Profesor adjunto en la Embry-Riddle Aeronautical University, EE.UU. Correo electrónico: flemingjs@earthlink.net

2 Psicólogo graduado de la Universidad Inca Garcilaso de la Vega. Jefe del servicio de Psicología de la Defensoría Municipal del Niño y del Adolescente. Correo electrónico: sikayax@yahoo.com.ar 



\section{Índices de simplicidad para ajuste de cargas factoriales}

¿Cómo uno puede evaluar la "bondad de ajuste" de la estructura en el análisis factorial exploratorio cuando el autor de una prueba desea obtener una estructura simple (en el sentido descrito)? Thurstone (1947) dio un número de criterios generales para definir una estructura simple de las cargas factoriales, pero frecuentemente los autores desean escribir "ítems puros" que demuestren lo que algunas veces se llama un conjunto de items con estructura simple. En tales casos, la matriz "ideal" de cargas factoriales toma la siguiente forma después de que las columnas de cargas han sido ordenadas:

$$
\begin{array}{lllll}
\mathrm{X} & 0 & 0 & \ldots & 0 \\
\mathrm{X} & 0 & 0 & \ldots & 0 \\
\mathrm{X} & 0 & 0 & \ldots & 0 \\
\cdot & & & \\
. & & & \\
0 & \mathrm{X} & 0 & \ldots & \\
0 & \mathrm{X} & 0 & \ldots & 0 \\
. & & & & \\
. & & & & \\
0 & 0 & 0 & \ldots X \\
0 & 0 & 0 & \ldots & \mathrm{X},
\end{array}
$$

... en donde cada variable tiene una carga no-cero en uno y solo un factor. En esta configuración, un " $X$ " indica una (elevada) carga saliente. Aunque es posible obtener un patrón como éste en el análisis factorial confirmatorio, es realmente imposible conseguir tal patrón en el análisis factorial exploratorio, pues habrán cargas 
muy pequeñas y estadísticamente insignificantes pero que diferirán de cero en pequeños montos debido al error aleatorio. Así que la matriz anterior representa el objetivo ideal para el autor de una prueba, en que cada variable se saturaría en uno y solo un factor, y los ítems que contribuyen a la complejidad de la estructura (es decir, que cargan en más de un factor) sean candidatos para eliminación.

Antes de avanzar con el artículo, debemos aclarar un punto: el término "carga" o "matriz de cargas factoriales" es claramente ambiguo (Harman, 1976; Thompson \& Daniel, 1996). En la situación ortogonal, la matriz de patrones (de los pesos de regresión) y de estructura (de correlaciones entre las variables manifiestas y las latentes) son idénticas. En el análisis oblicuo, sin embargo, hay muchas matrices de "cargas": el patrón primario, la estructura primaria, el patrón de referencia (que generalmente nunca se ha discutido) y la estructura de referencia (que es preferido por Thurstone, 1947, para la interpretación de factores). El patrón primario o la estructura de referencia (el último es proporcional al patrón primario) generalmente son simples en su estructura. Recomendamos interpretarlos en el análisis de ítems cuando se intente aproximarse a la matriz "ideal" de cargas factoriales. La matriz de estructura primaria, no obstante que es un adjunto útil para la interpretación de factores por derecho propio, nunca puede aparecer tan simple o tan cercana a la forma "ideal" como las otras matrices de "cargas". De este modo, en el presente artículo el término "cargas" se puede referir ya sea al patrón primario o a la estructura de referencia en el análisis oblicuo, o a la matriz de patrón/estructura en el análisis ortogonal. Pero observe que, si los coeficientes alfa se desean, se asumiría que es la matriz de patrón en el análisis oblicuo.

Siguiendo con el tema del artículo, se han utilizado varias medidas que pueden ser utilizadas para calcular la "bondad" de una 
solución en términos de su aproximación al ajuste ideal, basados en la simplicidad de las filas (el grado en que solo una variable satura sobre un factor), columnas (el grado de separación de cargas salientes de los no salientes) y en la matriz total. Estas medidas pueden suplementar otras medidas de desarrollo de escalas (unifactoriales) al seleccionar o refinar los ítems en escalas multifactoriales (ver por ejemplo, DeVellis, 1991, para la selección de ítems en escalas unifactoriales). Sin embargo, los índices presentados aquí también pueden ser de interés en la evaluación de la complejidad de soluciones factoriales en aplicaciones generales del análisis factorial, como se verá en uno de los ejemplos presentados en este artículo. Aunque los índices se han desarrollado en el contexto del análisis factorial exploratorio, también se pueden aplicar en el análisis factorial confirmatorio.

\section{Simplicidad de las filas}

Kaiser (1974) presentó un índice de simplicidad factorial $\left(\underline{\mathrm{ISF}}_{\mathrm{i}}\right)$, que se calcula para cada variable (ítem) $i$. Tal índice tiene un rango que va desde 0 (aunque los ítems debajo de .50 se consideran "inaceptables") hasta 1.0 (cuando todas menos una de las cargas tienen valor cero). Esta medida está basada en el criterio quartimax (Carroll, 1953; Saunders, 1953; Wrigley \& Neuhaus, 1954). También se puede calcular un ISF total. Sea $\underline{a}_{\mathrm{ij}}$ los elementos de la matriz A de patrón de los factores con $\underline{\mathrm{m}}$ filas (variables) y p columnas (factores). Luego, para la variable $i$ :

$$
I S F_{i}^{2}=\frac{p\left[\dot{Y}_{k} a_{i k}^{4}-\left(a_{i k}^{2}\right)\right]^{2}}{(p-1)\left(\begin{array}{ll}
\dot{Y}_{k} & a_{i k}^{2}
\end{array}\right)^{2}}
$$

Kaiser supuso que los valores ISF de .80 o más son "meritorios". 


\section{Simplicidad de las columnas}

Para evaluar la simplicidad de cada factor (columna), el Indice de Ajuste de la Escala $\left(\operatorname{IAE}_{\mathrm{j}}\right.$ ) para cada uno de los factores queda definido como:

$$
I A E_{j}=1-\frac{S C\left(N_{j}\right)}{S C\left(T_{j}\right)}
$$

... donde SC es la "suma de cuadrados" y $\mathrm{SC}\left(\underline{\underline{N}}_{\mathrm{j}}\right)$ es la suma de cuadrados de las cargas no salientes (de baja magnitud) y $\mathrm{SC}\left(\underline{\mathrm{T}}_{\mathrm{j}}\right)$ es la suma de cuadrados de todas las cargas, tanto salientes como no salientes, para la columna j. Esta medida también tiene un rango teórico de 0 hasta 1.0; el perfecto 1.0 se alcanza cuando todas las cargas no salientes son cero. Un valor de .50 se toma como que los ítems salientes no son mejores que los ítems no salientes; prácticamente hablando, los valores de al menos .80 son deseables, tal como en el caso del índice de Kaiser. Una medida global también se puede calcular para todas las columnas.

El cálculo de $\mathrm{IAE}_{\mathrm{j}}$ requiere algún criterio para diferenciar las cargas factoriales salientes de las no salientes. Para lograr ello, un método es predefinir un valor utilizando SIMLOAD (el parámetro LOVAL) para las cargas que se presumen que estarán en el hiperplano (por ejemplo, .15). Alternativamente, la distinción se puede hacer sobre una base más relativa, al determinar un punto de corte con el criterio Kaiser-Cerny (1978), que será discutida en la siguiente sección. Para fines de ordenamiento, el parámetro HIVAL está presente para las cargas que son salientes, tales como .45. De tal manera, los ítems arriba de HIVAL en un factor pero menos que LOVAL en los demás factores, son buenos candidatos para su retención en la escala. De acuerdo con un enfoque de análisis de ítems, no se puede asignar un ítem a más de un factor. 
Aunque ${\underline{\mathrm{IAE}_{\mathrm{j}}}}_{\mathrm{j}}$ es similar a un estadístico propuesto anteriormente, el Indice de Ajuste Factorial (Fleming, 1985), creemos que el presente índice tiene una mejor base racional. Paul Barrett (Barrett, 1996) definió una medida similar para el ajuste de escalas factoriales, que utiliza el valor absoluto de las cargas en vez de las cargas cuadradas. Sea $\underline{\mathrm{SC}}(\underline{\mathrm{S} j})$ la suma de cuadrados para las cargas salientes. La cantidad $\underline{\mathrm{SC}}(\underline{\mathrm{Sj}}) / \underline{\mathrm{SC}}(\underline{\mathrm{Nj}})$ es un cociente de tipo "signo al ruido", que será mayor en la medida que las cargas no salientes sean pequeñas. El $\underline{\mathrm{ISF}}_{\mathrm{j}}$ es un estadístico basado en este mismo concepto, pero el valor 1.0 está limitado a la situación en que todas las cargas no salientes son cero; ello es similar en su forma y lógica al coeficiente alfa (Cronbach, 1951).

\section{Simplicidad de la matriz (Global)}

Otro componente para la interpretación del ajuste es un estadístico introducido por Bentler (1977), que va desde 0 (estructura muy compleja) a 1.0 (estructura muy simple). Este índice es un tipo de varianza generalizada que se define como:

$$
\underline{S}=\left|D^{-1 / 2} B^{\prime} B D^{-1 / 2}\right|
$$

.... donde $\mathrm{B}$ es una matriz de cargas factoriales cuadradas, $\mathrm{D}=$ Diag (B'B). Si la matriz de cargas está en la forma presentada anteriormente como una matriz ideal, la matriz producto será una matriz de identidad con determinante 1.0. Bentler hizo notar que esto es una medida libre de escala, es decir, que no está afectado por algún escalamiento arbitrario de las columnas. Él describió también el uso de las rotaciones ortogonales y oblicuas (llamadas ortosim y oblisim, respectivamente) para maximizar este criterio. 
Las cargas en el hiperplano (es decir, las cargas esencialmente cero excepto por el error aleatorio) también pueden ser contadas como una medida de adecuabilidad en la aproximación a la estructura simple. Cuenta de hiperplanos (Cattell, 1952; Nunnally \& Bernstein, 1995) se describe como el número de cargas debajo de cierto punto de corte, pero esencialmente con valores cero. Este conteo se usa para ver cuántas cargas "cero" hay. En la construcción de pruebas, deberían haber cargas cercanas a cero en la matriz de cargas factoriales; estas cargas se consideran esencialmente cero entre $-.15 \mathrm{y}+.15$, de tal modo que se cuentan el número de tales valores en este rango.

En el método propuesto por Kaiser y Cerny (1978), en un trabajo que habla sobre la rotación oblicua, las cargas "cercanas a cero" son una función de la cantidad global de las cargas. En otras palabras, el criterio K-C "ajustará” el valor de cargas "no significativas" de acuerdo con la cantidad global de las cargas en el factor que está siendo considerado.

Para $\underline{\mathrm{m}}$ variables y $\mathrm{p}$ factores, el número de factores, $\underline{\mathrm{m}}(\underline{\mathrm{p}}-1)$ es el conteo ideal de hiperplanos. En el análisis exploratorio, es difícil estimar el error estándar de las cargas factoriales, así que generalmente se emplean reglas prácticas para identificar factores que son estadísticamente insignificantes en vez de criterios estadísticos. Para este propósito, SIMLOAD calcula el conteo de ítems que son menores que LOVAL en su valor absoluto (es decir, .15) y también computa el conteo de hiperplanos acorde con el criterio de Kaiser y Cerny (1978), basado en la función de poder absoluto de las cargas (Kendall \& Stuart, 1969). Para cada factor:

$$
f_{i}=\left(\frac{\left(\dot{Y}_{i}\left(a_{i j}^{2}\right)^{1 / b}\right)}{\underline{m}}\right)^{b / 2}
$$


Kaiser y Cerny sugirieron que $\underline{b}=4$ da el "mejor" de los resultados, aunque esto es un aporte que no deja de ser subjetivo. Las cargas menores que $\mathrm{f}_{\mathrm{j}}$, por lo tanto, se consideran ubicadas en el hiperplano. Esta función se adapta a la magnitud relativa de las cargas en cada factor: es decir, el criterio es grande para cargas altas, y pequeño para factores con cargas relativamente "bajas" (incrementar $\underline{b}$ hace que $\underline{f}_{j}$ sea pequeño, y viceversa).

Más que determinar un específico valor para LOVAL (como se ha discutido en la anterior sección), alternativamente se puede especificar por el procedimiento Kaiser-Cerny, separadamente para cada factor. En este caso, valores "cercanos a cero" para las cargas ordenadas serán diferentes para cada factor, dependiendo de la magnitud relativa de las cargas.

\section{Análisis adicionales para escalas factoriales}

Para facilitar el trabajo del investigador que está construyendo un instrumento, SIMLOAD también calcula el coeficiente alfa de Cronbach para los ítems componentes de las escalas, basada en datos estandarizados o directos. También calcula los efectos sobre el coeficiente alfa si cada ítem es removido. Estos estadísticos son opcionales y se calcularán si el usuario provee la matriz de correlaciones y desviaciones estándar (o matriz de covarianzas) junto con las cargas factoriales. Se asume que la matriz de cargas es una matriz de patrón en una métrica estandarizada (es decir, que no está escalada arbitrariamente).

El programa también calcula una matriz de intercorrelaciones entre las escalas (que no es lo mismo que la correlaciones entre puntajes factoriales o factores) y una matriz ordenada de cargas factoriales que reflejan la matriz "ideal" mostrada al inicio de este artículo. Para facilitar su interpretación, las cargas ordenadas menores a LOVAL son puestas como cero y las cargas definidas tienen asteriscos. 


\section{Ejemplos}

\section{Escala de Estrés en la Crianza}

El análisis se basó en la respuestas de 514 padres de familia varones y mujeres, provenientes de un colegio estatal (Merino, 2003). La solución factorial se mantuvo invariante entre varones y mujeres, y entre dos submuestras aleatoriamente extraídas para la inter validación de las escalas empíricamente halladas; se tomará por lo tanto la solución de la muestra total. Este ejemplo ilustrará el comportamiento de una escala en su construcción. La solución factorial provino de un análisis de ejes principales con rotación promax de las cargas; se mantuvo el parámetro kappa delta en 3.

El criterio Kaiser-Cerny para el conteo de valores hiperplanos, que está en función al tamaño relativo de los coeficientes de menor tamaño comparándolos con los de altos valores, obtiene un conteo de 17 (50\%). El estadístico de Bentler es .98. El conteo de hiperplanos es 7 (20.64\%), que mantiene una diferencia importante del conteo de hiperplanos ideal $(17,50 \%)$. El ISF en el nivel del ítem nos sugiere que hay ítems que son candidatos para eliminación: el ítem 6 (ISF $=.204$, "Actualmente no me siento muy contenta con él/ ella"), ítem 7 (ISF = .065, "Siento que no puedo manejarlo bien"), ítem 8 (ISF = .556, "Me siento cansada(o) o agotada(o) de corregirlo") e ítem 18 (ISF = .157, "La crianza de mi hijo(a) es una de las tareas más "pesadas" y difíciles que estoy haciendo"). La contribución de los ítems a la confiabilidad de su subescala parece no sugerir remoción de algún ítem (DeVellis, 1991). Las estimaciones de confiabilidad de las subescalas es .91 para Afectividad Negativa y .86 para Percepción de Ineficacia. En el Cuadro 1 se observa también que hay ítems con un ajuste ideal (ISF=1.0), como son los ítems 11, 13 y 14, aunque estos últimos alcanzan el máximo valor en el redondeo; sin incluir estos dos últimos, hay 7 ítems con ISF > .90. En contraste, tres ítems $(6,7,8$ y 18) revelan IFS que podrían evitarse en la estructura final de las subescalas. 
Medidas de simplicidad y de ajuste factorial

\section{Cuadro 1}

Cargas factoriales e índices de ajuste para la Escala de Estrés en la Crianza Disciplinaria

\begin{tabular}{|c|c|c|c|}
\hline & $\begin{array}{c}\text { Afectividad } \\
\text { Negativa }\end{array}$ & $\begin{array}{l}\text { Percepción de } \\
\text { Ineficacia }\end{array}$ & $\begin{array}{c}\text { Indices de } \\
\text { Simplicidad Factorial }\end{array}$ \\
\hline E1 & .053 & .704 & .98 \\
\hline E2 & .166 & .610 & .86 \\
\hline E3 & .092 & .539 & .94 \\
\hline $\mathrm{E} 4$ & .067 & .760 & .98 \\
\hline E5 & .101 & .627 & .94 \\
\hline E6 & .283 & .230 & .20 \\
\hline E7 & .364 & .341 & .06 \\
\hline E8 & .280 & .524 & .55 \\
\hline E9 & 612 & 249 & .71 \\
\hline E10 & .619 & .182 & .84 \\
\hline E11 & .760 & .010 & 1.0 \\
\hline E12 & .674 & .188 & .85 \\
\hline E13 & .765 & .034 & .99 \\
\hline E14 & .720 & .031 & .99 \\
\hline E16 & .519 & .260 & .59 \\
\hline E17 & .611 & .162 & .86 \\
\hline E18 & .297 & .348 & .15 \\
\hline $\begin{array}{c}\text { Indices de Ajuste } \\
\text { de la Escala } \\
\end{array}$ & 0.89 & 0.82 & $\begin{array}{c}\text { Total }=0.86 \\
(\text { mediana }=0.89)\end{array}$ \\
\hline $\begin{array}{c}\text { Indices de Simplicidad } \\
\text { Factorial Total }\end{array}$ & & & $\begin{array}{c}\text { Total }=0.928 \\
(\text { mediana }=0.970)\end{array}$ \\
\hline $\begin{array}{c}\text { Hiperplano } \\
\text { Kaiser - Cerny } \\
\end{array}$ & .36 & .28 & \\
\hline $\begin{array}{c}\text { Cargas en el } \\
\text { hiperplano } \\
{[-0.15,+0.15]}\end{array}$ & & & $7(20.6 \%)$ \\
\hline Correlaciones & $\begin{array}{c}\text { Afectividad } \\
\text { Negativa }\end{array}$ & $\begin{array}{c}\text { Percepción } \\
\text { Ineficacia }\end{array}$ & \\
\hline Afectividad Negativa & 1.0 & & \\
\hline Percepción Ineficacia & .77 & 1.0 & \\
\hline
\end{tabular}




\section{Factores en el WISC-R}

El Cuadro 2 muestra las cargas de patrón del análisis de las 11 escalas del WISC-R (Wechsler, 1974), para una muestra de 211 niños con problemas de aprendizaje, en que se extrajo tres factores rotados oblicuamente (oblimín directo) ("Verbal", "No Verbal" y "Distractibilidad"). Los datos fueron originalmente analizados por Tabachnick y Fleming (1986).

\section{Cuadro 2}

Matriz de patrón factorial para WISC-R, en una muestra de niños con discapacidades en el aprendizaje, y medidas de simplicidad asociadas

\begin{tabular}{|c|c|c|c|c|}
\hline Subprueba & $V C$ & $P O$ & $F D$ & $I S F$ \\
\hline 1. Información & 0.840 & -0.020 & -0.110 & .974 \\
\hline 2. Similaridades & 0.720 & 0.180 & -0.280 & .739 \\
\hline 3. Aritmética & 0.670 & -0.020 & 0.240 & .834 \\
\hline 4. Vocabulario & 0.840 & 0.000 & -0.070 & .990 \\
\hline 5. Comprensión & 0.740 & 0.140 & -0.050 & .942 \\
\hline 6. Memoria de Dígitos & 0.550 & -0.070 & 0.410 & .543 \\
\hline 7. Figuras Incompletas & 0.080 & 0.720 & -0.230 & .849 \\
\hline 8. Ordenamiento de Figuras & 0.020 & 0.560 & 0.170 & .875 \\
\hline 9. Diseño de Bloques & 0.120 & 0.690 & 0.160 & .884 \\
\hline 10. Rompecabezas & -0.060 & 0.810 & 0.000 & .992 \\
\hline IAE & .990 & .961 & .642 & \\
\hline
\end{tabular}

$\mathrm{CV}=$ Comprensión Verbal; $\mathrm{OP}=$ Orientación Perceptual; FD = Distractibilidad; $\mathrm{IAE}=$ Indice de Ajuste a la Escala; ISF = Indice de Simplicidad Factorial

Aunque esto no es un ejemplo de construcción de una prueba (se están analizando escalas más que ítems), aún es interesante ver dónde están las complejidades en la interpretación del WISC$\mathrm{R}$, y observar la conducta de varias medidas cuando dos de los factores exhiben una estructura simple, pero el tercero es más complejo; el ISF es inaceptablemente bajo para este factor. El ISF según Kaiser es al menos "meritorio" (> .80) para dos factores, 
pero es especialmente bajo para Memoria de Dígitos, que satura en dos factores. El ISF total es .903, el IAE es .917 y el índice de Bentler es .977. Aunque estos índices parecen aceptables, los valores individuales de ISF y IAE sugieren que hay espacio para mejorar la estructura. Si estos subtests fueran ítems, la remoción de los ítems podría ser justificada. El conteo de hiperplanos es como sigue: para el criterio igual a .15, 15 valores (45.5\%) están en tal hiperplano; para el criterio Kaiser-Cerny, 18 (54.5\%) están en el hiperplano; y el conteo "ideal" es 22 (66.7\%). Los coeficientes alfa estandarizados para Comprensión Verbal, Orientación Perceptual y Distractibilidad son, respectivamente .821, .687 y 0.0 (esto último es porque solamente la subescala Código y Símbolos define el tercer factor); los alfas desde los puntajes directos son muy similares. Si Memoria de Dígitos fuera removido, el alfa para CV se incrementaría solamente a .837.

\section{Importancia de medidas de simplicidad como herramienta general: conclusiones y recomendaciones}

Aunque hemos descrito este programa como una herramienta en los recursos del constructor de pruebas, estos índices pueden ser útiles para otros tipos de datos también. Por ejemplo, el segundo caso analizó los subtests del WISC-R en vez de ítems. Otra aplicación potencial es comparar la efectividad de varias rotaciones para tratar de producir la mejor estructura simple.

Este programa se diseñó como un adjunto para el análisis factorial exploratorio, pero los índices también pueden ser útiles en el análisis factorial confirmatorio - excepto que el conteo de hiperplanos debería estar basado en las cargas cero absolutas mas que en un valor arbitrario para definir las cargas "pequeñas" (es decir, definir LOVAL a cero). 
En esta línea, una interesante aplicación de los índices es su uso en la definición formal del grado de ajuste empírico de los ítems con la estructura hipotetizada, abordaje que es usual en el análisis factorial de grupo múltiple, que es un método confirmatorio de análisis factorial (Nunnally \& Bernstein, 1995). Este procedimiento podría añadir estadísticos descriptivos formales del grado en que los ítems se ajustan en los factores hipotetizados de antemano por el investigador y/o esperados teóricamente. La combinación de estos procedimientos, establecidos en un algoritmo de evaluación de la validez de constructo, ha sido presentada recientemente por Merino (2004); en tal estudio, se verificó la estructura interna de una escala de estilos de crianza y el grado de ajuste de los ítems a sus subescalas en términos de la complejidad factorial (Steinberg, Lamborn, Dornbusch \& Darling, 1992).

Los ejemplos ilustran sobre el comportamiento de los índices de simplicidad de patrones en aplicaciones típicas del desarrollo de escalas y análisis más generales. También nos dan una idea de cómo tales índices funcionan en la práctica. Las medidas globales (ISE, IAE y el estadístico de Bentler), cuando son considerados como medidas únicas, algunas veces pueden ser algo inapropiadas; éstas pueden tener valores altos aún cuando hay espacio para hacer mejoras, que es únicamente detectable al hacer una cuidadosa inspección de los índices individuales. Debido a las grandes ventajas que se obtienen con la inclusión de información cuantitativa para evaluar el ajuste de un factor, ellos pueden aplicados de manera rutinaria por los desarrolladores de instrumentos.

También puede ser útil e interesante la comparación de los índices de ajuste provenientes de diferentes muestras de sujetos. En tales casos el investigador puede comparar la variabilidad de la complejidad de los ítems aplicados en diferentes poblaciones de interés; por ejemplo, los ítems en una escala conductual respondidos 
por profesores y padres pueden originar diferencias en la complejidad de los factores.

Los índices de ajuste, en efecto, juegan un importante papel en la evaluación de la presencia de las amenazas a la validez, que son aspectos que merecen cuidadoso escrutinio por parte del que elabora pruebas. Downing (2002) señala que hay dos condiciones que son de preocupación al investigador: la varianza no relacionada al constructo y la deficiente representación del constructo. Un enfoque cuantitativo basado en medidas de ajuste puede ser un potencial recurso para evaluar estos aspectos indeseables en la elaboración y revisión de las pruebas psicológicas.

\section{Descripción del programa}

Lenguaje y sistema operativo: SIMLOAD se escribió en Visual Fortran 6.5.0 y compilado bajo Windows 98. SIMLOAD es un programa interactivo que corre en las últimas versiones de Windows.

Datos de ingreso (Input): el programa pedirá un título, la matriz de cargas factoriales (patrón), etiquetas de las variables (opcional), parámetros para HIVAL (criterio para definir cargas salientes; por ejemplo, .35) y LOVAL (criterio para cargas en el hiperplano; por ejemplo, .15). Alternativamente, el criterio de hiperplanos de Kaiser-Cerny puede ser determinado para definir las cargas factoriales bajas. Si se desea coeficientes alfa de Cronbach para las escalas, el usuario debe proveer también una matriz de correlaciones (o matriz de covarianza) y desviaciones estándar. En un mismo archivo el programa lee toda esta información.

Resultados (Output): el resultado incluye las medidas de simplicidad de filas (Kaiser, 1974), de columnas (Fleming \& Merino; este 
artículo), y de la matriz (Bentler, 1977; y el conteo de hiperplanos descrito anteriormente). Adicionalmente, se puede obtener coeficientes alfa como una ayuda complementaria para el investigador, junto con el efecto sobre alfa si se remueve cada ítem. El programa también da las cargas ordenadas originalmente y en orden. En el último caso, las cargas en el hiperplano se imprimen como ceros y aquellos arriba de HIVAL aparecen con un asterisco para facilitar la interpretación. Para la interpretación, la matriz de cargas ordenadas debería ser comparada con la matriz "ideal" inicial.

Disponibilidad: SIMLOAD está disponible solicitándolo al autor primario del presente documento, James Fleming, en: 2275 Pine Drive, Prescott, AZ, 86306, USA. Informar sobre la dirección electrónica si se desea bajarlo desde Internet. Correspondencia sobre este artículo o programa también dirigirla a: flemingjs@earthlink.net.

\section{Referencias}

Arndt, S. \& Merino, C. (2003). Un enfoque confirmatorio en la construcción y revisión de escalas: análisis factorial de grupo múltiple. Manuscrito presentado para su publicación.

Barrett, P. (1996). An evaluation of the psychometric properties of the concept 5.2 Occupational Personality Questionnaire. Journal of Occupational and Organizational Psychology, 69, 1-19.

Bentler, P. M. (1977). Factor simplicity index and transformations. Psychometrika, 42, 277-295.

Carroll, J. B. (1953). An analytical solution for approximating simple structure in factor analysis. Psychometrika, 18, 23-38.

Cattell, R. B. (1952). Factor analysis: An introduction and manual for the psychologist and social scientist. Nueva York: Harper. 
Cronbach, L. J. (1951). Coefficient alpha and the internal structure of tests. Psychometrika, 16, 297-334.

DeVellis, R. F. (1991). Scale development: Theory and applications. Newbury Park, CA: Sage.

Downing, S. M. (2002). Threats to the validity of locally developed multiple-choice tests in medical education: Construct-irrelevant variance and construct underrepresentation. Advances in Health Sciences Education, 7, 235-241.

Fleming, J. S. (1985). An index of fit for factor scales. Educational and Psychological Measurement, 45, 725-728.

Harman, H. H. (1976). Modern factor analysis (3a. ed.). Chicago: University of Chicago Press.

Kaiser, H. F. (1974). An index of factorial simplicity. Psychometrika, 39, 31-35.

Kaiser, H. F. \& Cerny, B. A. (1978). Casey's method for fitting hyperplanes from an intermediate orthomax criterion. Multivariate Behavioral Research, 13, 395-401.

Kendall, M. G. \& Stuart, A. (1969). The advanced theory of statistics (3a. ed., Vol. 1). Nueva York: Hafner.

Merino, C. (2003). Escala de Estrés en la Crianza. Manuscrito no publicado. Defensoría Municipal del Niño y del Adolescente, Lima.

Merino, C. (2004). Análisis factorial confirmatorio y confiabilidad de la Escala de Estilos de Crianza de L. Steinberg. Tesis de licenciatura, Universidad Inca Garcilaso de la Vega, Lima.

Merino, C. \& Fleming, J. S. (2005). Validación factorial preliminar de una escala de estrés en la crianza. Manuscrito en preparación.

Nunnally, J. C. \& Bernstein, I. J. (1995). Teoría psicométrica (3 ${ }^{\mathrm{a}}$. ed.). México, D. F: McGraw-Hill.

Saunders, D. R. (1953). An analytic method for rotation to orthogonal simple structure [Resumen]. American Psychologist, 8, 428.

Steinberg, L., Lamborn, S., Dornbusch, S. \& Darling, N. (1992). Impact of parenting practices on adolescent achievement: 
Authoritative parenting, school involvement, encouragement to succeed. Child Development, 63, 1266-1281.

Tabachnick, B. G. \& Fleming, J. S. (1986, abril). WISC-R profile and factor analyses of learning disabled subgroups. Documento presentado en la reunión anual de la Western Psychological Association, Seattle, Washington, EE.UU.

Thompson, B. \& Daniel, L. G. (1996). Factor analytic evidence for the construct validity of scores: An historical overview and some guidelines. Educational and Psychological Measurement, 45, 203-209.

Thurstone, L. L. (1947). Multiple factor analysis. Chicago: University of Chicago Press.

Wechsler, D. (1974). Manual for the Wechsler Intelligence Scale for Children - Revised. San Antonio, TX: Psychological Corporation.

Wrigley, C. \& Neuhaus, J. O. (1954). The quartimax method: An analytic approach to orthogonal simple structure. British Journal of Statistical Psychology, 7, 81-91. 